\title{
Longitudinal complex magnetic susceptibility and relaxation time of superparamagnetic particles with cubic magnetic anisotropy
}

\author{
Yuri P. Kalmykov and Sergey V. Titov \\ Institute of Radio Engineering and Electronics of the Russian Academy of Sciences, Vvedenskii, Square 1, Fryazino, Moscow \\ Region 141120, Russian Federation \\ William T. Coffey* \\ Department of Electronic and Electrical Engineering, Trinity College, Dublin 2, Ireland
}

(Received 3 February 1998)

\begin{abstract}
The magnetic relaxation of single-domain ferromagnetic particles with cubic magnetic anisotropy is treated by averaging the Gilbert-Langevin equation for an individual particle, so that the system of linear differentialrecurrence relations for the appropriate equilibrium correlation functions is derived without recourse to the Fokker-Planck equation. The solution of this system (in terms of matrix continued fractions) is determined and the longitudinal relaxation time and spectrum of the complex magnetic susceptibility are evaluated. It is shown that in contrast to particles with uniaxial anisotropy, there is an inherent geometric dependence of the complex susceptibility and the relaxation time on the damping parameter arising from coupling of longitudinal and transverse relaxation modes. [S0163-1829(98)06829-5]
\end{abstract}

\section{INTRODUCTION}

A single-domain ferromagnetic particle is characterized by an internal potential, which has several local states of equilibrium with potential barriers between them. If the particles are small $(\sim 100 \AA)$ so that the potential barriers are relatively low, the magnetization vector $\mathbf{M}$ may cross over the barriers between one potential well and another due to thermal agitation. ${ }^{1}$ The ensuing thermal instability of magnetization results in the phenomenon of superparamagnetism (so-called). ${ }^{2}$ The thermal fluctuations and relaxation of the magnetization of a single-domain particle currently merit attention in view of their importance in the context of magnetic recording media ${ }^{3}$ and paleomagnetism. ${ }^{4}$

For the purpose of mathematical simplification, particles with uniaxial magnetic anisotropy have for the most part been considered (see, for example, Refs. 2 and 5-12). Although the use of the uniaxial potential considerably simplifies the analysis, the results obtained by means of this approximation have, however, a restricted area of applicability. ${ }^{13}$ For more realistic anisotropy potentials, such as a cubic potential, either the discrete orientation approximation was used, or only asymptotes of the continuous diffusion model were derived (see, for example, Refs. 2 and 13-19). Both approaches, however, do not apply to the most interesting case, where the magnetic anisotropy energy is comparable to the thermal energy $k T$.

The magnetization dynamics of single-domain particles for the continuous diffusion model is similar to rotation of a Brownian particle in a liquid and obeys the Fokker-Planck equation for the probability density distribution $W(\{\mathbf{u}\}, t) .^{2,20,21}$ The Fokker-Planck equation is derived from Gilbert's equation ${ }^{2,20}$ with a random white noise field, which takes into account the thermal fluctuations of the magnetization of an individual particle. In general, for magnetic relaxation of a system of single-domain particles with cubic anisotropy, the Fokker-Planck equation can be formally solved, for example, by means of an expansion of the distribution function $W$ in spherical harmonics. ${ }^{14,19}$ In such an approach the problem is reduced to the solution of an infinite system of linear differential-recurrence relations for the averaged spherical harmonics (moments):

$$
\frac{d}{d t} \mathbf{X}(t)=\mathbf{A} \mathbf{X}(t)
$$

where $\mathbf{X}(t)$ is the column vector consisting of the system moments and $\mathbf{A}$ is the infinite system matrix. The elements of this matrix depend on certain parameters characterizing the anisotropy energy and dissipation. [The detailed derivation of Eq. (1) was given recently by Geoghegan et al. $\left.{ }^{19}\right]$ The numerical solution of Eq. (1) (eigenvalues and eigenvectors of the system matrix) may be obtained by consecutive increase of the number of the equations $N$ until convergence is attained. Such an approach to cubic anisotropy has been used, for example, by Aharoni and Eisenstein in Refs. 14, 16, and 17, where several low-order eigenvalues were evaluated numerically in the intermediate damping limit. Unfortunately, the application of this direct matrix approach to the present problem is inconvenient, especially for low damping and intermediate barrier heights (where asymptotic estimates are unreliable) as it is necessary to carry out calculations for $N \sim 10^{3}-10^{4}$ and convergence of the solution is consequently difficult to achieve. Furthermore, the complex magnetic susceptibility spectrum for the cubic anisotropy in the context of the continuous diffusion model has not been calculated as yet either for high or low damping.

On account of the difficulties encountered in the numerical analysis, which we have mentioned, the cubic case is to some extent incomplete. ${ }^{2}$ This gap in our knowledge of the solution may, however, be bridged if one uses a matrix continued fraction method for the solution of infinite systems of recurrence relations developed in Refs. 22 and 23. Such a method was applied by us for particles with the simplest 
axially symmetric anisotropy in Refs. 9 and 12 . Here, the method is extended to obtain the longitudinal component of the magnetic susceptibility $\chi_{\|}(\omega)$ and the relaxation time $\tau_{\|}$ of a system of noninteracting single-domain particles with cubic anisotropy. Our calculations of $\chi_{\|}(\omega)$ and $\tau_{\|}$are based on linear-response theory. ${ }^{22,23}$ In order to evaluate the $\chi_{\|}(\omega)$ and $\tau_{\|}$we must first calculate the equilibrium correlation function $C_{\|}(t)$ of the magnetization, which is more conveniently accomplished directly from Gilbert's equation ${ }^{24}$ rather than from the Fokker-Planck equation. ${ }^{12}$ Thus, we bypass the Fokker-Planck equation entirely. The behavior of $\chi_{\|}(\omega)$ is investigated for all ranges of the anisotropy energy $(\sigma)$ and dissipation $(\alpha)$ parameters. The results obtained are compared both with previously available solutions and asymptotic estimates based on the Kramers escape rate theory.

\section{DERIVATION OF DIFFERENTIAL-RECURRENCE RELATIONS FROM GILBERT'S EQUATION AUGMENTED BY A RANDOM FIELD TERM} is ${ }^{6}$

Gilbert's equation ${ }^{24}$ in the presence of thermal agitation

$$
\frac{d}{d t} \mathbf{M}(t)=\gamma\{\mathbf{M}(t) \times[\mathbf{H}(t)+\mathbf{h}(t)-\eta \dot{\mathbf{M}}(t)]\},
$$

where $\gamma$ is the gyromagnetic ratio, $\eta$ is the damping parameter, $\mathbf{H}(t)$ is the magnetic field acting on the particle, which may consist of externally applied magnetic fields and the crystalline anisotropy field, and a random Gaussian white noise field $\mathbf{h}(t)$, which has the properties

$$
\begin{gathered}
\overline{h_{i}(t)}=0, \\
\overline{h_{i}(t) h_{j}\left(t^{\prime}\right)}=(2 k T \eta / \nu) \delta_{i j} \delta\left(t-t^{\prime}\right) .
\end{gathered}
$$

$v$ is the volume of the particle. Here the overbar means the statistical average over an ensemble of particles that all have at time $t$ the same magnetization $\mathbf{M}$. If $V(\{\mathbf{M}\})$ is the free energy per unit volume expressed as a function of components of $\mathbf{M}$, then

$$
\mathbf{H}=-\frac{\partial}{\partial \mathbf{M}} V(\{\mathbf{M}\}),
$$

where

$$
\frac{\partial}{\partial \mathbf{M}}=\mathbf{i} \frac{\partial}{\partial M_{x}}+\mathbf{j} \frac{\partial}{\partial M_{y}}+\mathbf{k} \frac{\partial}{\partial M_{z}} .
$$

$M_{x}=M_{s} \sin \vartheta \cos \varphi=M_{s} u_{x}, M_{y}=M_{s} \sin \vartheta \sin \varphi=M_{s} u_{y}$, $M_{z}=M_{s} \cos \vartheta=M_{s} u_{z}, M_{s}$ is the saturation magnetization, and $\vartheta, \varphi$ are the polar and azimuthal angles, respectively. It is assumed throughout this analysis that the magnetization is uniform inside the particle and only the orientation and not the magnitude of the magnetization is subject to variations. The assumptions made in the derivation of Gilbert's equation were discussed elsewhere (e.g., Ref. 13).

We shall use the representation for the free energy of unit volume of a particle possessing cubic magnetic anisotropy, ${ }^{2,23}$ viz.,

$$
V=K\left(u_{x}^{2} u_{y}^{2}+u_{x}^{2} u_{z}^{2}+u_{y}^{2} u_{z}^{2}\right)=\frac{K}{4}\left(\sin ^{4} \vartheta \sin ^{2} 2 \varphi+\sin ^{2} 2 \vartheta\right),
$$

where $K$ is the anisotropy constant, which may have both positive and negative values. For $K>0$, the potential (4) has 6 minima, 8 maxima, and 12 saddle points (for example, in the directions [100], [111], and [110], accordingly). ${ }^{2}$ If $K$ $<0$, the maxima and minima are interchanged.

Gilbert's equation (2) may be rearranged explicitly using the properties of the vector triple product formula to yield ${ }^{2}$

$$
\begin{aligned}
\frac{d}{d t} \mathbf{M}(t)= & M_{s} g^{\prime}\{\mathbf{M}(t) \times[\mathbf{H}(t)+\mathbf{h}(t)]\}+h^{\prime}(\{\mathbf{M}(t) \\
& \times[\mathbf{H}(t)+\mathbf{h}(t)]\} \times \mathbf{M}(t)),
\end{aligned}
$$

where

$$
g^{\prime}=\frac{\gamma}{\left(1+\alpha^{2}\right) M_{s}}, \quad h^{\prime}=\frac{\gamma \alpha}{\left(1+\alpha^{2}\right) M_{s}}=\alpha g^{\prime}
$$

and the dimensionless damping constant $\alpha$ is given by

$$
\alpha=\gamma \eta M_{s} .
$$

Equation (5) is of the same mathematical form as the Landau-Lifshitz equation ${ }^{5}$ except that both of the parameters $g^{\prime}$ and $h^{\prime}$ depend on $\alpha$.

Expanding Eq. (5) in its Cartesian components we have

$$
\begin{aligned}
\frac{1}{h^{\prime} M_{s}} \frac{d}{d t} u_{x}(t)= & {\left[1-u_{x}^{2}(t)\right] h_{x}(t)-\left[\alpha^{-1} u_{z}(t)+u_{x}(t) u_{y}(t)\right] h_{y}(t)+\left[\alpha^{-1} u_{y}(t)-u_{z}(t) u_{x}(t)\right] h_{z}(t)+\left[1-u_{x}^{2}(t)\right] H_{x}(t) } \\
& -\left[\alpha^{-1} u_{z}(t)+u_{x}(t) u_{y}(t)\right] H_{y}(t)+\left[\alpha^{-1} u_{y}(t)-u_{z}(t) u_{x}(t)\right] H_{z}(t)
\end{aligned}
$$

$$
\begin{aligned}
\frac{1}{h^{\prime} M_{s}} \frac{d}{d t} u_{y}(t)= & {\left[\alpha^{-1} u_{z}(t)-u_{x}(t) u_{y}(t)\right] h_{x}(t)+\left[1-u_{y}^{2}(t)\right] h_{y}(t)-\left[\alpha^{-1} u_{x}(t)+u_{y}(t) u_{x}(t)\right] h_{z}(t) } \\
& +\left[\alpha^{-1} u_{z}(t)-u_{x}(t) u_{y}(t)\right] H_{x}(t)+\left[1-u_{y}^{2}(t)\right] H_{y}(t)-\left[\alpha^{-1} u_{x}(t)+u_{y}(t) u_{x}(t)\right] H_{z}(t),
\end{aligned}
$$$$
\frac{1}{h^{\prime} M_{s}} \frac{d}{d t} u_{z}(t)=-\left[\alpha^{-1} u_{y}(t)+u_{x}(t) u_{z}(t)\right] h_{x}(t)+\left[\alpha^{-1} u_{x}(t)-u_{y}(t) u_{z}(t)\right] h_{y}(t)+\left[1-u_{z}^{2}(t)\right] h_{z}(t)
$$$$
-\left[\alpha^{-1} u_{y}(t)+u_{x}(t) u_{z}(t)\right] H_{x}(t)+\left[\alpha^{-1} u_{x}(t)-u_{y}(t) u_{z}(t)\right] H_{y}(t)+\left[1-u_{z}^{2}(t)\right] H_{z}(t) .
$$ 
The set of stochastic differential equations (7)-(9) contains multiplicative noise terms $h_{i}(t) u_{j}(t) u_{k}(t)$. They pose an interpretation problem for these equations as has been discussed in Refs. 22 and 23. We recall, taking the set of the Langevin equations for the $N$ stochastic variables $\{\xi(t)\}$ $=\left\{\xi_{1}(t), \xi_{2}(t), \ldots, \xi_{N}(t)\right\}$,

$\frac{d \xi_{i}(t)}{d t}=h_{i}(\{\xi(t)\}, t)+g_{i j}(\{\xi(t)\}, t) \Gamma_{j}(t) \quad(i, j=1, \ldots, N)$,

with

$$
\overline{\Gamma_{i}(t)}=0, \quad \overline{\Gamma_{i}(t) \Gamma_{j}\left(t^{\prime}\right)}=2 D \delta_{i j} \delta\left(t-t^{\prime}\right),
$$

and interpreting them as Stratonovich equations, that the averaged equations for the sharp values $\xi_{i}(t)=x_{i}$ at time $t$ $\operatorname{are}^{22,23}$

$$
\begin{aligned}
\frac{d x_{i}}{d t}= & \lim _{\tau \rightarrow 0} \frac{\overline{\xi_{i}(t+\tau)-x_{i}}}{\tau}=h_{i}(\{\mathbf{x}\}, t) \\
& +D g_{k j}(\{\mathbf{x}\}, t) \frac{\partial}{\partial x_{k}} g_{i j}(\{\mathbf{x}\}, t) \quad(i, j=1, \ldots, N),
\end{aligned}
$$

where $\xi_{i}(t+\tau)(\tau>0)$ is the solution of Eq. (10) with the initial conditions $\xi_{i}(t)=x_{i}$. In Eqs. (10) and (11) summation over $j$ and $k$ is understood (Einstein's notation). The proof of Eq. (11) can be found elsewhere (see Ref. 22, pp. 54 and 55). We remark that we use the Stratonovich definition for the average of the multiplicative noise term here because that definition is the mathematical idealization of the physical relaxation process. ${ }^{24}$ Thus, it is unnecessary to transform the Langevin equations (7)-(9) to Itô equations (e.g., Ref. 22). Moreover, one can apply the methods of ordinary analysis if the Stratonovich definition is used. ${ }^{22}$

In like manner one can prove ${ }^{12}$ that the averaged (according to the Stratonovich definition ${ }^{25}$ ) equation for an arbitrary differentiable function $f(\{\xi\})$ has the following form:

$$
\begin{aligned}
\frac{d f(\{\mathbf{x}\})}{d t}= & \lim _{\tau \rightarrow 0} \frac{\overline{f(\{\xi(t+\tau)\})-f(\{\mathbf{x}\})}}{\tau} \\
= & h_{i}(\{\mathbf{x}\}, t) \frac{\partial}{\partial x_{i}} f(\{\mathbf{x}\})+D g_{k j}(\{\mathbf{x}\}, t) \frac{\partial}{\partial x_{k}} \\
& \times\left[g_{i j}(\{\mathbf{x}\}, t) \frac{\partial}{\partial x_{i}} f(\{\mathbf{x}\})\right],
\end{aligned}
$$

where summation over $i, j$, and $k$ is understood.

In the study of the magnetic relaxation, the quantities of interest are averages involving the normalized spherical harmonics $Y_{n, m}$ defined as

$$
Y_{n, m}=(-1)^{m}\left(\frac{(2 n+1)(n-m) !}{4 \pi(n+m) !}\right)^{1 / 2} e^{i m \varphi}\left(1-\cos ^{2} \vartheta\right)^{m / 2} \frac{d^{m} P_{n}(\cos \vartheta)}{d \cos ^{m} \vartheta}, \quad Y_{n,-m}=(-1)^{m} Y_{n, m}^{*}, \quad m>0,
$$

where the $P_{n}(x)$ are the Legendre polynomials ${ }^{26}$ and the asterisk denotes the complex conjugate. The $Y_{n, m}$ are expressed in terms of $u_{x}, u_{y}, u_{z}$ as follows:

$$
Y_{n, m}=(-1)^{m}\left(\frac{(2 n+1)(n-m) !}{4 \pi(n+m) !}\right)^{1 / 2}\left(u_{x}+i u_{y}\right)^{m} \frac{d^{m} P_{n}\left(u_{z}\right)}{d u_{z}^{m}}
$$

Noting that according to the Stratonovich definition the conventional rules of transformation of a stochastic variable (ordinary calculus) may be used, ${ }^{25}$ we can obtain from Eqs. (7), (8), and (9) the stochastic differential equation of motion for $Y_{n, m}$ :

$$
\begin{aligned}
\dot{Y}_{n, m}\left(u_{x}(t), u_{y}(t), u_{z}(t)\right)= & (-1)^{m}\left(\frac{(2 n+1)(n-m) !}{4 \pi(n+m) !}\right)^{1 / 2}\left\{m\left[\dot{u}_{x}(t)+i \dot{u}_{y}(t)\right]\left[u_{x}(t)+i u_{y}(t)\right]^{m-1} \frac{d^{m} P_{n}\left[u_{z}(t)\right]}{d u_{z}^{m}}\right. \\
& \left.+\dot{u}_{z}(t)\left[u_{x}(t)+i u_{y}(t)\right]^{m} \frac{d^{m+1} P_{n}\left[u_{z}(t)\right]}{d u_{z}^{m+1}}\right\} .
\end{aligned}
$$

Further on averaging Eq. (13) and using the theorem of Eq. (12), we obtain after tedious algebra

$$
\begin{aligned}
\tau_{N} \dot{Y}_{n, m}\left(u_{x}, u_{y}, u_{z}\right)= & \sum_{s=-1} \sum_{r=-4}^{4} d_{n, m, r, s} \\
& \times Y_{n+r, m+4 s}\left(u_{x}, u_{y}, u_{z}\right),
\end{aligned}
$$

where $d_{n, m, r,-s}=d_{n,-m, r, s}^{*}$ (the $d_{n, m, r, s}$ are listed in Appen$\operatorname{dix}$ A). The details of the derivation of such systems of equa- tions may be found elsewhere. ${ }^{19,23,27}$ We remark that all the $Y_{n, m}$ in Eq. (14) are functions of $u_{x}, u_{y}, u_{z}$. Also $u_{x}, u_{y}, u_{z}$ in Eq. (14) and $u_{x}(t), u_{y}(t), u_{z}(t)$ in the stochastic Eqs. (7)(9) and (13) have different meanings, namely, $u_{x}(t), u_{y}(t), u_{z}(t)$ in Eqs. (7)-(9) and (13) are stochastic variables (processes) while $u_{x}, u_{y}, u_{z}$ in Eq. (14) are the sharp (definite) values $u_{k}(t)=u_{k}$ at time $t$. Instead of using different symbols for the two quantities, we have distinguished sharp values at time $t$ from the stochastic variables by deleting the time argument as in Refs. 22 and 23 . 
The quantities $Y_{n, m}$ in Eq. (14) are functions of the sharp values $u_{k}$, which are themselves random variables with distribution (probability density) function $W$. In the absence of external perturbations, the system is in equilibrium with Boltzmann distribution function $W_{0}$ given by

$$
W_{0}(\vartheta, \varphi)=\frac{1}{Z} \exp \left[-\frac{\nu V(\vartheta, \varphi)}{k T}\right],
$$

where $Z$ is the partition function. We may now construct from Eq. (14) an infinite hierarchy of differential-recurrence relations for any desired equilibrium correlation functions. In particular, upon multiplying Eq. (14) by $\cos \vartheta(0)$, and averaging the resulting equation over the equilibrium distribution function $W_{0}$ at the instant $t=0$, we obtain

$$
\tau_{N} \frac{d}{d t} c_{n, m}(t)=\sum_{s=-1}^{1} \sum_{r=-4}^{4} d_{n, m, r, s} c_{n+r, m+4 s}(t),
$$

where

$$
c_{n, m}(t)=\left\langle\cos \vartheta(0) Y_{n, m}(t)\right\rangle_{0}
$$

are the equilibrium correlation functions,

$$
\tau_{N}=\frac{\nu}{2 k T h^{\prime}}=\frac{\nu M_{s}\left(1+\alpha^{2}\right)}{2 k T \gamma \alpha} .
$$

is the diffusion relaxation time, \langle\rangle$_{0}$ designates the equilibrium average at $t=0$ defined as

$$
\langle A\rangle_{0}=\int_{0}^{2 \pi} \int_{0}^{\pi} A(\vartheta, \varphi) W_{0}(\vartheta, \varphi) \sin \vartheta d \vartheta d \varphi .
$$

We remark that Eq. (15) may also be derived from the corresponding Fokker-Planck equation, ${ }^{19}$

$$
\begin{aligned}
2 \tau_{N} \frac{\partial W}{\partial t}= & \frac{1}{\sin \vartheta} \frac{\partial}{\partial \vartheta}\left\{\operatorname { s i n } \vartheta \left[\frac{\nu}{k T}\left(\frac{\partial V}{\partial \vartheta}-\frac{1}{\alpha \sin \vartheta} \frac{\partial V}{\partial \phi}\right)\right.\right. \\
& \left.\left.\times W+\frac{\partial W}{\partial \vartheta}\right]\right\}+\frac{1}{\sin \vartheta} \frac{\partial}{\partial \phi}\left[\frac { \nu } { k T } \left(\frac{1}{\alpha} \frac{\partial V}{\partial \vartheta}\right.\right. \\
& \left.\left.+\frac{1}{\sin \vartheta} \frac{\partial V}{\partial \phi}\right) W+\frac{1}{\sin \vartheta} \frac{\partial W}{\partial \phi}\right] .
\end{aligned}
$$

\section{EVALUATION OF THE COMPLEX SUSCEPTIBILITY IN TERMS OF MATRIX CONTINUED FRACTIONS}

Before proceeding, we must first summarize the principal results of linear-response theory [Ref. 22, Chap. 7]. The application of this theory to magnetic problems predicts that the decay of the longitudinal component of the magnetization $\left\langle M_{\|}\right\rangle(t)$ of a system of noninteracting single-domain ferromagnetic particles, when a small constant external field $\mathbf{H}_{1}\left[\nu(\mathbf{M} \cdot \mathbf{H})_{1} / k T \ll 1\right]$ applied along the $z$ axis (which is at the easy axis of the particle for $K>0$ ) has been switched off at time $t=0$, is

$$
\left\langle M_{\|}\right\rangle(t)=\chi_{\|} H_{1} C_{\|}(t),
$$

where

$$
\chi_{\|}=\frac{N_{0} \nu^{2} M_{s}^{2}}{k T}\left(\frac{4 \pi}{3}\right)^{1 / 2} c_{1,0}(0)=\frac{N_{0} \nu^{2} M_{s}^{2}}{3 k T}
$$

is the longitudinal static magnetic susceptibility, $N_{0}$ is the number of particles per unit volume, and

$$
C_{\|}(t)=\frac{c_{1,0}(t)}{c_{1,0}(0)}
$$

is the normalized autocorrelation function of the longitudinal component of the magnetization of the particle. The longitudinal component of the complex magnetic susceptibility $\chi_{\|}(\omega)$ is

$$
\chi_{\|}(\omega)=\chi_{\|}^{\prime}(\omega)-i \chi_{\|}^{\prime \prime}(\omega)=\chi_{\|}\left\{1-i \omega \widetilde{C}_{\|}(i \omega)\right\},
$$

where

$$
\tilde{C}_{\|}(i \omega)=\int_{0}^{\infty} C_{\|}(t) e^{-i \omega t} d t
$$

Another quantity of interest is the integral relaxation (or correlation) time $\tau_{\|}$, which is defined as the area under $C_{\|}(t)$ :

$$
\tau_{\|}=\int_{0}^{\infty} C_{\|}(t) d t=\tilde{C}_{\|}(0) .
$$

The $\tau_{\|}$may equivalently be defined in the context of the Fokker-Planck equation (18) converted to the SturmLiouville problem as

$$
\tau_{\|}=\frac{\sum_{k} C_{k} / \lambda_{k}}{\sum_{k} C_{k}},
$$

where $\lambda_{k}$ and $C_{k}$ are the eigenvalues and their corresponding weight coefficients (amplitudes), and the correlation function $C_{\|}(t)$ is given by

$$
C_{\|}(t)=\sum_{k} C_{k} e^{-i \lambda_{k} t}
$$

It is usually impossible to evaluate $\tau_{\|}$and $C_{\|}(t)$ analytically from Eq. (22) as a knowledge of the law of formation of the eigenvalues and their corresponding weights (amplitudes) is required. The approach we shall use below does not attempt to calculate $\tau_{\|}$and $\widetilde{C}_{\|}(i \omega)$ by explicitly calculating the eigenvalue spectrum as required by Eq. (22), rather it yields $\tau_{\|}$ and $\tilde{C}_{\|}(i \omega)$ in terms of matrix continued fractions.

The formal matrix continued fraction approach to the solution of the recurrence equations such as Eq. (15), where two indexes vary, was described in Ref. 22. However, in practice it is rather inconvenient, as one must use [as in the initial equation (1)] matrices of infinite dimension. We shall use below a more sophisticated method of solution of Eq. (15), so that it is possible to reduce the computational task to operations involving matrices of finite dimensions.

Thus we introduce a column vector $\mathbf{C}_{n}(t)$ : 


$$
\mathbf{C}_{n}(t)=\left(\begin{array}{c}
\mathbf{c}_{4 n}(t) \\
\mathbf{c}_{4 n-1}(t) \\
\mathbf{c}_{4 n-2}(t) \\
\mathbf{c}_{4 n-3}(t)
\end{array}\right) .
$$

This vector consist of four column subvectors $\mathbf{c}_{4 n-i}(t)$, which are given by

$$
\mathbf{c}_{4 n-i}(t)=\left(\begin{array}{c}
c_{4 n-i,-4\left(n-1+\delta_{i 0}\right)}(t) \\
c_{4 n-i,-4\left(n-2+\delta_{i 0}\right)}(t) \\
\vdots \\
c_{4 n-i, 4\left(n-1+\delta_{i 0}\right)}(t)
\end{array}\right), \quad i=0,1,2,3 .
$$

The vector $\mathbf{C}_{n}(t)$ has $8 n-2$ elements. Thus, Eq. (15) can be transformed into the matrix three-term differentialrecurrence relation

$$
\begin{gathered}
\tau_{N} \frac{d}{d t} \mathbf{C}_{n}(t)=\mathbf{Q}_{n}^{-} \mathbf{C}_{n-1}(t)+\mathbf{Q}_{n} \mathbf{C}_{n}(t)+\mathbf{Q}_{n}^{+} \mathbf{C}_{n+1}(t), \\
n=1,2,3, \ldots
\end{gathered}
$$

with

$$
\mathbf{C}_{0}(t)=\mathbf{0} \quad \text { and } \quad \mathbf{C}_{1}(t)=\left(\begin{array}{c}
c_{4,-4}(t) \\
c_{4,0}(t) \\
c_{4,4}(t) \\
c_{3,0}(t) \\
c_{2,0}(t) \\
c_{1,0}(t)
\end{array}\right) .
$$

The matrices $\mathbf{Q}_{n}, \mathbf{Q}_{n}^{+}, \mathbf{Q}_{n}^{-}$in Eq. (25) are given by

$$
\begin{gathered}
\mathbf{Q}_{n}=\left(\begin{array}{cccc}
\mathbf{A}_{4 n} & \mathbf{B}_{4 n} & \mathbf{P}_{4 n} & \mathbf{D}_{4 n} \\
\mathbf{E}_{4 n-1} & \mathbf{A}_{4 n-1} & \mathbf{B}_{4 n-1} & \mathbf{P}_{4 n-1} \\
\mathbf{F}_{4 n-2} & \mathbf{E}_{4 n-2} & \mathbf{A}_{4 n-2} & \mathbf{B}_{4 n-2} \\
\mathbf{G}_{4 n-3} & \mathbf{F}_{4 n-3} & \mathbf{E}_{4 n-3} & \mathbf{A}_{4 n-3}
\end{array}\right), \\
\mathbf{Q}_{n}^{+}=\left(\begin{array}{cccc}
\mathbf{H}_{4 n} & \mathbf{G}_{4 n} & \mathbf{F}_{4 n} & \mathbf{E}_{4 n} \\
\mathbf{0} & \mathbf{H}_{4 n-1} & \mathbf{G}_{4 n-1} & \mathbf{F}_{4 n-1} \\
\mathbf{0} & \mathbf{0} & \mathbf{H}_{4 n-2} & \mathbf{G}_{4 n-2} \\
\mathbf{0} & \mathbf{0} & \mathbf{0} & \mathbf{H}_{4 n-3}
\end{array}\right), \\
\mathbf{Q}_{n}^{-}=\left(\begin{array}{cccc}
\mathbf{J}_{4 n} & \mathbf{0} & \mathbf{0} & \mathbf{0} \\
\mathbf{D}_{4 n-1} & \mathbf{J}_{4 n-1} & \mathbf{0} & \mathbf{0} \\
\mathbf{P}_{4 n-2} & \mathbf{D}_{4 n-2} & \mathbf{J}_{4 n-2} & \mathbf{0} \\
\mathbf{B}_{4 n-3} & \mathbf{P}_{4 n-3} & \mathbf{D}_{4 n-3} & \mathbf{J}_{4 n-3}
\end{array}\right) .
\end{gathered}
$$

The matrices $\mathbf{Q}_{n}, \mathbf{Q}_{n}^{+}, \mathbf{Q}_{n}^{-}$consist of the three-diagonal submatrices $\mathbf{A}, \mathbf{B}, \mathbf{E}, \mathbf{D}, \mathbf{F}, \mathbf{G}, \mathbf{H}, \mathbf{J}$, and $\mathbf{P}$, which are described in detail in Appendix A. The dimensions of $\mathbf{Q}_{n}, \mathbf{Q}_{n}^{+}, \mathbf{Q}_{n}^{-}$are $(8 n-2) \times(8 n-2), \quad(8 n-2) \times(8 n+6), \quad$ and $(8 n-2)$ $\times(8 n-10)$, respectively. The exception is

$$
\mathbf{Q}_{1}^{-}=\left(\begin{array}{c}
\mathbf{J}_{4} \\
0 \\
0 \\
0
\end{array}\right),
$$

which degenerates to a column vector of dimension 6 .
On applying the general method of solution of the matrix three-term differential-recurrence relations, Eq. (25), suggested in Ref. 23, we obtain the exact solution for the Laplace transform $\widetilde{\mathbf{C}}_{1}(s)$ in terms of matrix continued fractions, viz.,

$$
\begin{aligned}
\widetilde{\mathbf{C}}_{1}(s)= & \tau_{N}\left[\tau_{N} s \mathbf{I}-\mathbf{Q}_{1}-\mathbf{Q}_{1}^{+} \mathbf{S}_{2}(s)\right]^{-1}\left\{\mathbf{C}_{1}(0)\right. \\
& \left.+\sum_{n=2}^{\infty}\left[\prod_{k=2}^{n} \mathbf{Q}_{k-1}^{+} \mathbf{S}_{k}(s)\left(\mathbf{Q}_{k}^{-}\right)^{-1}\right] \mathbf{C}_{n}(0)\right\},
\end{aligned}
$$

where $\mathbf{I}$ is the identity matrix, and the matrix continued fraction $\mathbf{S}_{n}(s)$ is defined as

$$
\mathbf{S}_{n}(s)=\left[\tau_{N} s \mathbf{I}-\mathbf{Q}_{n}-\mathbf{Q}_{n}^{+} \mathbf{S}_{n+1}(s)\right]^{-1} \mathbf{Q}_{n}^{-} .
$$

The initial condition vectors $\mathbf{C}_{n}(0)$ may also be evaluated in terms of matrix continued fractions. ${ }^{22,23}$ The initial values $c_{n, m}(0)$ in Eq. (15) are given by

$$
\begin{aligned}
c_{n, m}(0)= & \left\langle\cos \vartheta(0) Y_{n, m}(0)\right\rangle_{0} \\
= & \left(\frac{(n+1)^{2}-m^{2}}{(2 n+1)(2 n+3)}\right)^{1 / 2}\left\langle Y_{n+1, m}\right\rangle_{0} \\
& +\left(\frac{n^{2}-m^{2}}{(2 n+1)(2 n-1)}\right)^{1 / 2}\left\langle Y_{n-1, m}\right\rangle_{0} .
\end{aligned}
$$

According to Eq. (14), the equilibrium averages $\left\langle Y_{n, m}\right\rangle_{0}$ satisfy the recurrence relation ${ }^{19}$

$$
\sum_{s=-1}^{1} \sum_{r=-4}^{4} d_{n, m, r, s}\left\langle Y_{n+r, m+4 s}\right\rangle_{0}=0,
$$

which may be written as the three-term matrix recurrence relation:

$$
\mathbf{Q}_{n}^{-} \mathbf{R}_{n-1}+\mathbf{Q}_{n} \mathbf{R}_{n}+\mathbf{Q}_{n}^{+} \mathbf{R}_{n+1}=\mathbf{0}, \quad n=1,2,3, \ldots,
$$

where

$$
\mathbf{R}_{n}=\left(\begin{array}{c}
\mathbf{r}_{4 n} \\
\mathbf{r}_{4 n-1} \\
\mathbf{r}_{4 n-2} \\
\mathbf{r}_{4 n-3}
\end{array}\right), \quad \mathbf{R}_{0}=\frac{1}{\sqrt{4 \pi}} .
$$

The column subvector $\mathbf{r}_{4 n-i}$ is

$$
\mathbf{r}_{4 n-i}=\left(\begin{array}{c}
\left\langle Y_{4 n-i,-4\left(n-1+\delta_{i 0}\right)}\right\rangle_{0} \\
\left\langle Y_{4 n-i,-4\left(n-2+\delta_{i 0}\right)}\right\rangle_{0} \\
\vdots \\
\left\langle Y_{4 n-i, 4\left(n-1+\delta_{i 0}\right)}\right\rangle_{0}
\end{array}\right), \quad i=0,1,2,3 .
$$

The solution of Eq. (35) has the following form:

$$
\mathbf{R}_{n}=\mathbf{S}_{n}(0) \mathbf{R}_{n-1}=\mathbf{S}_{n}(0) \mathbf{S}_{n-1}(0) \cdots \mathbf{S}_{2}(0) \mathbf{S}_{1}(0) / \sqrt{4 \pi},
$$

where

$$
\mathbf{S}_{n}(0)=\left[-\mathbf{Q}_{n}-\mathbf{Q}_{n}^{+} \mathbf{S}_{n+1}(0)\right]^{-1} \mathbf{Q}_{n}^{-} .
$$

Thus the $\mathbf{C}_{n}(0)$ are given by 


$$
\mathbf{C}_{n}(0)=\mathbf{K}_{n}^{-} \mathbf{R}_{n-1}+\mathbf{K}_{n} \mathbf{R}_{n}+\mathbf{K}_{n}^{+} \mathbf{R}_{n+1}, \quad n=1,2,3 \ldots,
$$

where

$$
\begin{gathered}
\mathbf{K}_{n}=\left(\begin{array}{ccccc}
\mathbf{0} & \mathbf{U}_{4 n} & \mathbf{0} & \mathbf{0} \\
\mathbf{V}_{4 n-1} & \mathbf{0} & \mathbf{U}_{4 n-1} & \mathbf{0} \\
\mathbf{0} & \mathbf{V}_{4 n-2} & \mathbf{0} & \mathbf{U}_{4 n-2} \\
\mathbf{0} & \mathbf{0} & \mathbf{V}_{4 n-3} & \mathbf{0}
\end{array}\right), \\
\mathbf{K}_{n}^{+}=\left(\begin{array}{cccc}
\mathbf{0} & \mathbf{0} & \mathbf{0} & \mathbf{V}_{4 n} \\
\mathbf{0} & \mathbf{0} & \mathbf{0} & \mathbf{0} \\
\mathbf{0} & \mathbf{0} & \mathbf{0} & \mathbf{0} \\
\mathbf{0} & \mathbf{0} & \mathbf{0} & \mathbf{0}
\end{array}\right) \\
\mathbf{K}_{n}^{-}=\left(\begin{array}{cccc}
\mathbf{0} & \mathbf{0} & \mathbf{0} & \mathbf{0} \\
\mathbf{0} & \mathbf{0} & \mathbf{0} & \mathbf{0} \\
\mathbf{0} & \mathbf{0} & \mathbf{0} & \mathbf{0} \\
\mathbf{U}_{4 n-3} & \mathbf{0} & \mathbf{0} & \mathbf{0}
\end{array}\right)
\end{gathered}
$$

with the submatrix $\mathbf{K}_{1}^{-}$degenerating to a column vector of dimension 6. The matrices $\mathbf{K}_{n}, \mathbf{K}_{n}^{+}, \mathbf{K}_{n}^{-}$are comprised of submatrices $\mathbf{U}$ and $\mathbf{V}$, which are explicitly listed in Appendix B.

Taking account of Eq. (38), Eq. (40) yields

$$
\begin{aligned}
\mathbf{C}_{n}(0)= & \frac{1}{\sqrt{4 \pi}}\left\{\mathbf{K}_{n}^{-}+\left[\mathbf{K}_{n}\right.\right. \\
& \left.\left.+\mathbf{K}_{n}^{+} \mathbf{S}_{n+1}(0)\right] \mathbf{S}_{n}(0)\right\} \mathbf{S}_{n-1}(0) \cdots \mathbf{S}_{1}(0) .
\end{aligned}
$$

In particular,

$$
\mathbf{C}_{1}(0)=\frac{1}{\sqrt{4 \pi}}\left\{\mathbf{K}_{1}^{-}+\left[\mathbf{K}_{1}+\mathbf{K}_{1}^{+} \mathbf{S}_{2}(0)\right] \mathbf{S}_{1}(0)\right\}
$$

The exact matrix continued fraction solution [Eqs. (31) and (42)] we have obtained is very convenient for the purpose of computation. All the matrix continued fractions and series involved converge very rapidly, thus 10-12 downward iterations in calculating the continued fractions and 11-14 terms in the series (31) are enough to arrive at an accuracy of not less than six significant digits in the majority of cases. The greatest dimension of all the matrices involved is of the order $10^{2}$, which allows one to carry out the calculations on a personal computer.

\section{RESULTS AND DISCUSSIONS}

The behavior of the relaxation time $\tau_{\|}$as a function of the dimensionless parameter $\sigma=\nu K / 4 k T$ for various values of the damping parameter $\alpha$ is shown in Fig. 1 [for $K>0$ both the saddle energy and the potential barrier height are equal to $\sigma$, whereas for $K<0$ the barrier height $=|\sigma| / 3$ while the saddle energy $=-|\sigma|$ (Ref. 2)]. In this figure the results of the calculation from the exact formulas are compared with those evaluated from the asymptotic formulas for $\tau_{\|}$in the low temperature or high barrier limit $(|\sigma| \gg 1)$ for various ranges of $\alpha$. The asymptotic formulas in the intermediate to high damping (IHD) limit $(\alpha \geqslant 1)$ were obtained in Refs. 2 and 15

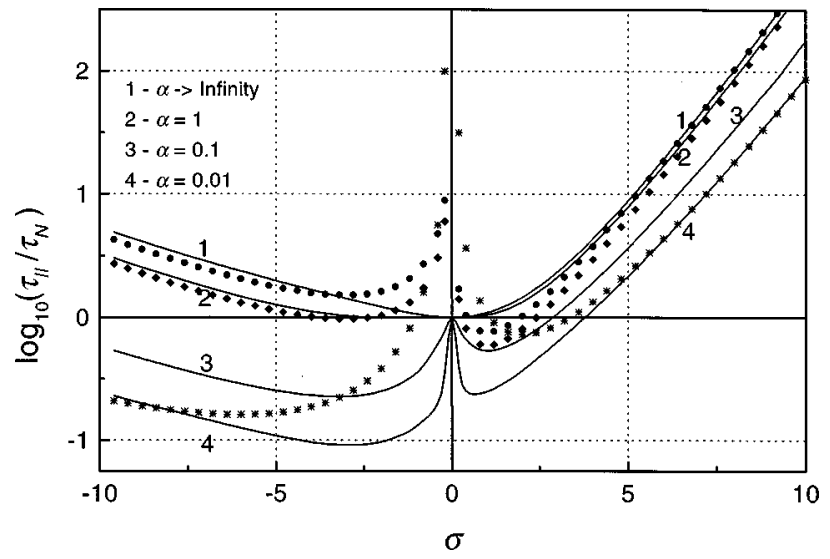

FIG. 1. $\log _{10}\left(\tau / \tau_{N}\right)$ vs $\sigma$ for various values of the damping parameter $\alpha$. Solid lines are the numerical calculations from Eq. (22) at $\alpha \rightarrow \infty$ (curve 1), 1 (2), 0.1 (3), and 0.01 (4). Filled circles and squares are the calculation from Eqs. (44) and (45) for $\alpha \rightarrow \infty$ and 1, respectively; crosses are the calculation from Eqs. (46) and (47) at $\alpha=0.01$.

and reviewed in Ref. 19. They can be written as (in our notation)

$$
\begin{gathered}
\tau_{\|} \sim \frac{\tau_{N} \pi e^{\sigma}}{2 \sqrt{2} \sigma\left(\sqrt{9+8 / \alpha^{2}}+1\right)}, \quad \sigma>0 \\
\tau_{\|} \sim \frac{3 \tau_{N} \pi e^{|\sigma| / 3}}{2 \sqrt{2}|\sigma|\left(\sqrt{9+8 / \alpha^{2}}-1\right)}, \quad \sigma<0 .
\end{gathered}
$$

In the opposite, low damping limit $(\alpha \ll 1)$ the appropriate asymptotic solution was given by Klik and Gunther ${ }^{13,18}$ and reviewed by Coffey. ${ }^{28}$ Their formulas applied to the present problem yield

$$
\begin{gathered}
\tau_{\|} \sim \frac{\pi k T e^{\sigma}}{2 \omega_{A} \Delta E} \approx \frac{\tau_{N} \pi e^{\sigma}}{8 \sigma^{2}}, \quad \sigma>0 \\
\tau_{\|} \sim \frac{2 \pi k T e^{|\sigma| / 3}}{3 \omega_{A} \Delta E} \approx \frac{\tau_{N} \pi e^{|\sigma| / 3}}{4 \sigma^{2}}, \quad \sigma<0
\end{gathered}
$$

where

$$
\omega_{A}=\left\{\begin{array}{cl}
\frac{8 \sigma \gamma k T}{\nu M_{s}} & (\sigma>0) \\
\frac{16|\sigma| \gamma k T}{3 \nu M_{s}} & (\sigma<0)
\end{array}\right.
$$

is the frequency of oscillation in the potential well, and

$$
\Delta E=\alpha \oint_{\nu V=E_{0}}\left[\left(1-u_{z}^{2}\right) \frac{\partial}{\partial \varphi} \nu V d \varphi-\frac{1}{1-u_{z}^{2}} \frac{\partial}{\partial u_{z}} \nu V d u_{z}\right]
$$

is the energy loss per cycle of the almost periodic motion at the saddle point energy $E_{0}$. Here instead of the numerical evaluation of the integral in Eq. (48) (which is of the order of the barrier height ${ }^{13}$ ) we have used an approximation

$$
\Delta E \approx \alpha\left|E_{0}\right|=\alpha \nu|K| / 4
$$




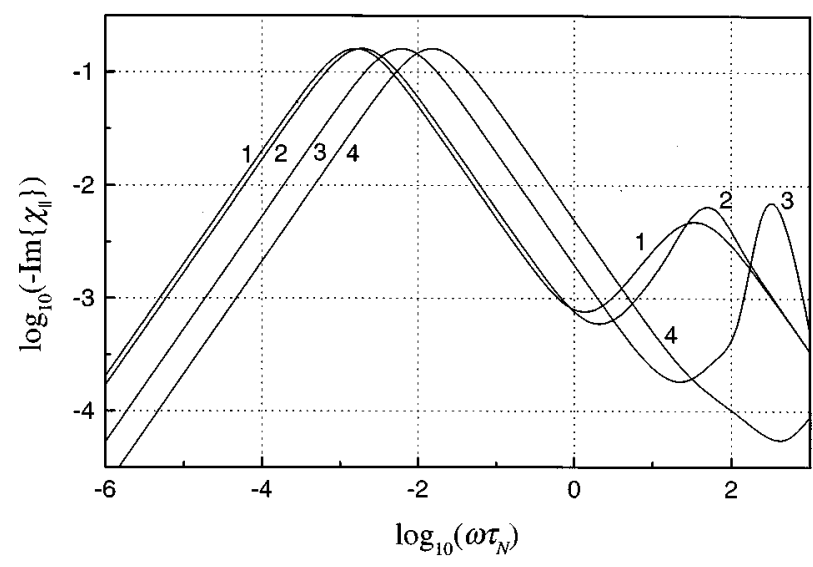

FIG. 2. $\log _{10}\left(\chi_{\|}^{\prime \prime}\right)$ vs $\log _{10}\left(\omega \tau_{N}\right)$ for $\sigma=10$ and $\alpha \rightarrow \infty$ (curve 1), $\alpha=1$ (curve 2), $\alpha=0.1$ (curve 3), and $\alpha=0.01$ (curve 4).

for both positive and negative values of the anisotropy constant. Experimental and theoretical estimations of $\alpha$ are discussed, for example, in Refs. 6, 13, and 29. These estimations give values of $\alpha$ of the order $0.01-0.1{ }^{6,13}$ It should be noted that $\tau_{N}$ in Eqs. (44)-(47) depends on $\alpha$ as defined by Eq. (17).

As far as a physical interpretation is concerned, the relaxation time $\tau_{\|}$is determined by the slowest low-frequency relaxation mode, which governs transitions of the magnetization vector over the barriers from one potential well into another. The characteristic frequency of this overbarrier relaxation mode is determined by the inverse of the smallest nonvanishing eigenvalue $\lambda_{1}$ of the Fokker-Planck equation (18). As one can see in Fig. 1, in contrast to uniaxial anisotropy (see, for example, Refs. 6, 9, and 10), $\tau_{\|} / \tau_{N}$ for particles with cubic anisotropy strongly depends on the damping parameter $\alpha$. It should be noted that for crossover values of $\alpha$ $(\sim \alpha \approx 0.1)$ neither the IHD formulas (44) and (45) nor the low damping Eqs. (46) and (47) yield reliable quantitative estimates. Thus a more detailed analysis is necessary. ${ }^{13}$ The dependence on the damping parameter is shown also in the spectra of the imaginary part of the complex susceptibility $\chi_{\|}^{\prime \prime}(\omega)$, shown in Fig. 2. In Fig. 3, the spectrum of $\chi_{\|}^{\prime \prime}(\omega)$ is plotted for $\alpha=0.1$ and various values of $\sigma$. (The calculations were carried out with $\nu^{2} M_{s}^{2} N_{0} / k T=1$.) In these figures two

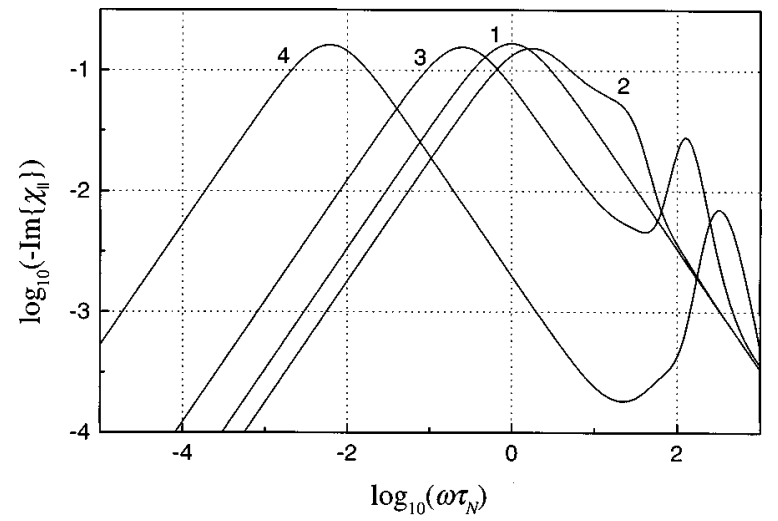

FIG. 3. $\log _{10}\left(\chi_{\|}^{\prime \prime}\right)$ vs $\log _{10}\left(\omega \tau_{N}\right)$ for $\alpha=0.1$ and $\sigma=0$ (curve 1), $\sigma=1$ (curve 2), $\sigma=5$ (curve 3), and $\sigma=10$ (curve 4).

peaks in the loss spectrum are visible. The first (lowfrequency) peak is located at frequencies of the order of the average frequency of reorientation of the magnetization vector. The characteristic frequency and the half-width of this low-frequency band are determined by $\tau_{\|}$. The second, much weaker peak is caused by the contribution of the highfrequency intrawell and transverse relaxation modes.

Thus, the longitudinal component of the magnetic susceptibility $\chi_{\|}(\omega)$ and relaxation time $\tau_{\|}$of systems of singledomain particles with cubic magnetic anisotropy are given by the exact equation (31) formulated in terms of matrix continued fractions. In contrast to the uniaxial anisotropy, 6,9 where the damping only enters in the diffusion time [Eq. (17)], there is an inherent geometric dependence of $\chi_{\|}(\omega)$ and $\tau_{\|} / \tau_{N}$ for the particles with cubic magnetic anisotropy on the value of the damping parameter $\alpha$ arising from coupling of the longitudinal and transverse relaxation modes. In the derivation of the above results, it was supposed that all particles are identical. In order to take into account the polydispersity of the particles of a real sample, one must also average the susceptibility over appropriate distribution functions (e.g., over that of particle volumes).

\section{ACKNOWLEDGMENTS}

The support of the work by the International Association for the Promotion of Cooperation with Scientists from the New Independent States of the Former Soviet Union (Grant No. INTAS 96-0663) is gratefully acknowledged.

\section{APPENDIX A: EXPLICIT REPRESENTATION OF SUBMATRICES AND THEIR ELEMENTS IN EQS. (27)-(29)}

There are three kinds of submatrices in Eqs. (27)-(29). The submatrices $\mathbf{A}_{4 n}, \mathbf{E}_{4 n}, \mathbf{F}_{4 n}, \mathbf{G}_{4 n}, \mathbf{A}_{4 n-1}, \mathbf{A}_{4 n-2}, \mathbf{A}_{4 n-3}$, $\mathbf{B}_{4 n-1}, \mathbf{B}_{4 n-2}, \mathbf{B}_{4 n-3}, \mathbf{D}_{4 n-1}, \mathbf{E}_{4 n-2}, \mathbf{E}_{4 n-3}, \mathbf{F}_{4 n-3}, \mathbf{P}_{4 n-1}, \mathbf{P}_{4 n-2}$ have the form

$$
\mathbf{X}_{4 n-i}=\left(\begin{array}{ccclc}
x_{4 n-i,-4\left(n-1+\delta_{i 0}\right)} & x_{4 n-i,-4\left(n-1+\delta_{i 0}\right)}^{+} & 0 & \cdots & 0 \\
x_{4 n-i,-4\left(n-2+\delta_{i 0}\right)}^{-} & x_{4 n-i,-4\left(n-2+\delta_{i 0}\right)} & x_{4 n-i,-4\left(n-2+\delta_{i 0}\right)}^{+} & \cdots & 0 \\
0 & x_{4 n-i,-4\left(n-3+\delta_{i 0}\right)}^{-} & x_{4 n-i,-4\left(n-3+\delta_{i 0}\right)} & \cdots & 0 \\
\vdots & \vdots & \vdots & \ddots & \vdots \\
0 & 0 & 0 & \cdots & x_{4 n-i, 4\left(n-1+\delta_{i 0}\right)}
\end{array}\right)
$$

$(i=0,1,2,3)$ and have dimension $\left[2\left(n+\delta_{0 i}\right)-1\right] \times\left[2\left(n+\delta_{0 i}\right)-1\right]$. The submatrices $\mathbf{B}_{4 n}, \mathbf{D}_{4 n}, \mathbf{J}_{4 n}, \mathbf{P}_{4 n}, \mathbf{D}_{4 n-2}, \mathbf{D}_{4 n-3}$, $\mathbf{J}_{4 n-1}, \mathbf{J}_{4 n-2}, \mathbf{J}_{4 n-3}, \mathbf{P}_{4 n-3}$ are defined as 


$$
\mathbf{X}_{4 n-i}=\left(\begin{array}{ccccc}
x_{4 n-i,-4\left(n-1+\delta_{i 0}\right)}^{+} & 0 & 0 & \cdots & 0 \\
x_{4 n-i,-4\left(n-2+\delta_{i 0}\right)} & x_{4 n-i,-4\left(n-2+\delta_{i 0}\right)}^{+} & 0 & \cdots & 0 \\
x_{4 n-i,-4\left(n-3+\delta_{i 0}\right)}^{-} & x_{4 n-i,-4\left(n-3+\delta_{i 0}\right)} & x_{4 n-i,-4\left(n-3+\delta_{i 0}\right)}^{+} & \cdots & 0 \\
\vdots & \vdots & \vdots & \ddots & \vdots \\
0 & 0 & 0 & \cdots & x_{4 n-i, 4\left(n-1+\delta_{i 0}\right)}^{-}
\end{array}\right)
$$

$(i=0,1,2,3)$ and have dimension $\left[2\left(n+\delta_{0 i}\right)-1\right] \times\left[2\left(n+\delta_{0 i}\right)-3\right]$. The submatrices $\mathbf{H}_{4 n}, \mathbf{E}_{4 n-n}, \mathbf{F}_{4 n-1}, \mathbf{F}_{4 n-2}, \mathbf{G}_{4 n-1}$, $\mathbf{G}_{4 n-2}, \mathbf{G}_{4 n-3}, \mathbf{H}_{4 n-1}, \mathbf{H}_{4 n-2}, \mathbf{H}_{4 n-3}$ are given by

$$
\mathbf{X}_{4 n-i}=\left(\begin{array}{ccccc}
x_{4 n-i,-4\left(n-1+\delta_{i 0}\right)}^{-} & x_{4 n-i,-4\left(n-1+\delta_{i 0}\right)} & x_{4 n-i,-4\left(n-1+\delta_{i 0}\right)}^{+} & \cdots & 0 \\
0 & x_{4 n-i,-4\left(n-2+\delta_{i 0}\right)}^{-} & x_{4 n-i,-4\left(n-2+\delta_{i 0}\right)} & \cdots & 0 \\
0 & 0 & x_{4 n-i,-4\left(n-3+\delta_{i 0}\right)}^{-} & \cdots & 0 \\
\vdots & \vdots & \vdots & \ddots & \vdots \\
0 & 0 & 0 & \cdots & x_{4 n-i, 4\left(n-1+\delta_{i 0}\right)}^{+}
\end{array}\right)
$$

and have the dimension $\left[2\left(n+\delta_{0 i}\right)-1\right] \times\left[2\left(n+\delta_{0 i}\right)+1\right]$. The submatrix elements are

$$
\begin{aligned}
& a_{n, m}=d_{n, m, 0,0}=\sigma \frac{9(n-1) n(n+1)(n+2)-15 m^{2}\left[6 n(n+1)-5-7 m^{2}\right]}{(2 n-3)(2 n-1)(2 n+3)(2 n+5)}-\frac{n(n+1)}{2}, \\
& a_{n, m}^{-}=a_{n,-m}^{+}=d_{n, m, 0,-1}=\frac{15 \sigma \sqrt{(n+m)(n-m+4)\left[n^{2}-(m-3)^{2}\right]\left[n^{2}-(m-2)^{2}\right]\left[n^{2}-(m-1)^{2}\right]}}{2(2 n-3)(2 n-1)(2 n+3)(2 n+5)}, \\
& b_{n, m}=d_{n, m-1,0}=-\frac{3 i \sigma m\left(3 n^{2}-5-7 m^{2}\right)}{\alpha\left(4 n^{2}-9\right)}\left(\frac{n^{2}-m^{2}}{4 n^{2}-1}\right)^{1 / 2}, \\
& b_{n, m}^{-}=-b_{n,-m}^{+}=d_{n, m-1,-1}=-\frac{3 i \sigma}{2 \alpha\left(4 n^{2}-9\right)}\left(\frac{(n+m-4)(n+m)\left[n^{2}-(m-3)^{2}\right]\left[n^{2}-(m-2)^{2}\right]\left[n^{2}-(m-1)^{2}\right]}{4 n^{2}-1}\right)^{1 / 2}, \\
& p_{n, m}=d_{n, m,-2,0}=\frac{\sigma(2 n+9)\left(n^{2}-n-2-7 m^{2}\right)}{(2 n-5)(2 n-1)(2 n+3)}\left(\frac{\left[n^{2}-m^{2}\right]\left[(n-1)^{2}-m^{2}\right]}{(2 n+1)(2 n-3)}\right)^{1 / 2}, \\
& p_{n, m}^{-}=p_{n,-m}^{+}=d_{n, m,-2,-1}=-\frac{\sigma(2 n+9)}{2(2 n-5)(2 n-1)(2 n+3)} \\
& \times\left(\frac{(n+m-5)(n+m-4)(n+m-3)(n+m)\left[n^{2}-(m-2)^{2}\right]\left[n^{2}-(m-1)^{2}\right]}{(2 n+1)(2 n-3)}\right)^{1 / 2}, \\
& d_{n, m}=d_{n, m,-3,0}=-\frac{7 i \sigma m}{\alpha(2 n-3)(2 n-1)}\left(\frac{\left(n^{2}-m^{2}\right)\left[(n-1)^{2}-m^{2}\right]\left[(n-2)^{2}-m^{2}\right]}{(2 n-5)(2 n+1)}\right)^{1 / 2}, \\
& d_{n, m}^{-}=-d_{n,-m}^{+}=d_{n, m,-3,-1}=\frac{i \sigma}{2 \alpha(2 n-3)(2 n-1)}\left(\frac{(n+m-6)(n+m-5) \cdots(n+m-1)(n+m)(n-m+1)}{(2 n-5)(2 n+1)}\right)^{1 / 2}, \\
& e_{n, m}=d_{n, m, 1,0}=-\frac{i 3 \sigma m\left(3 n^{2}+6 n-2-7 m^{2}\right)}{\alpha(2 n-1)(2 n+5)}\left(\frac{\left[(n+1)^{2}-m^{2}\right]}{(2 n+1)(2 n+3)}\right)^{1 / 2} \\
& e_{n, m}^{-}=-e_{n,-m}^{+}=d_{n, m, 1,-1}=\frac{i 3 \sigma}{2 \alpha(2 n-1)(2 n+5)} \\
& \times\left(\frac{(n+m)(n-m+3)(n-m+4)(n-m+5)\left[n^{2}-(m-1)^{2}\right]\left[n^{2}-(m-2)^{2}\right]}{(2 n+1)(2 n+3)}\right)^{1 / 2}, \\
& f_{n, m}=d_{n, m, 2,0}=-\frac{\sigma(2 n-7)\left(n^{2}+3 n-7 m^{2}\right)}{(2 n-1)(2 n+3)(2 n+7)}\left(\frac{\left[(n+1)^{2}-m^{2}\right]\left[(n+2)^{2}-m^{2}\right]}{(2 n+1)(2 n+5)}\right)^{1 / 2},
\end{aligned}
$$




$$
\begin{aligned}
& f_{n, m}^{-}=f_{n,-m}^{+}=d_{n, m, 2,-1}=\frac{\sigma(2 n-7)}{2(2 n-1)(2 n+3)(2 n+7)} \\
& \times\left(\frac{(n+m)\left[n^{2}-(m-1)^{2}\right](n+m-2)(n-m+3)(n-m+4)(n-m+5)(n-m+6)}{(2 n+1)(2 n+5)}\right)^{1 / 2}, \\
& g_{n, m}=d_{n, m, 3,0}=-\frac{7 i \sigma m}{\alpha(2 n+3)(2 n+5)}\left(\frac{\left[(n+3)^{2}-m^{2}\right]\left[(n+2)^{2}-m^{2}\right]\left[(n+1)^{2}-m^{2}\right]}{(2 n+1)(2 n+7)}\right)^{1 / 2}, \\
& g_{n, m}^{-}=-g_{n,-m}^{+}=d_{n, m, 3,-1}=-\frac{i \sigma}{2 \alpha(2 n+3)(2 n+5)}\left(\frac{(n+m)(n-m+1)(n-m+2) \cdots(n-m+7)}{(2 n+1)(2 n+7)}\right)^{1 / 2}, \\
& h_{n, m}=d_{n, m, 4,0}=-\frac{7 \sigma n}{(2 n+3)(2 n+5)(2 n+7)}\left(\frac{\left[(n+1)^{2}-m^{2}\right]\left[(n+2)^{2}-m^{2}\right]\left[(n+3)^{2}-m^{2}\right]\left[(n+4)^{2}-m^{2}\right]}{(2 n+1)(2 n+9)}\right)^{1 / 2}, \\
& h_{n, m}^{-}=h_{n,-m}^{+}=d_{n, m, 4,-1}=-\frac{\sigma n}{2(2 n+3)(2 n+5)(2 n+7)}\left(\frac{(n-m+1)(n-m+2) \cdots(n-m+7)(n-m+8)}{(2 n+1)(2 n+9)}\right)^{1 / 2}, \\
& j_{n, m}=d_{n, m,-4,0}=\frac{7 \sigma(n+1)}{(2 n-5)(2 n-3)(2 n-1)}\left(\frac{\left[(n-3)^{2}-m^{2}\right]\left[(n-2)^{2}-m^{2}\right]\left[(n-1)^{2}-m^{2}\right]\left[n^{2}-m^{2}\right]}{(2 n-7)(2 n+1)}\right)^{1 / 2}, \\
& j_{n, m}^{-}=j_{n,-m}^{+}=d_{n, m,-4,-1}=\frac{\sigma(n+1)}{2(2 n-5)(2 n-3)(2 n-1)}\left(\frac{(n+m-7)(n+m-6) \cdots(n+m-1)(n+m)}{(2 n-7)(2 n+1)}\right)^{1 / 2},
\end{aligned}
$$

where

$$
\sigma=\frac{\nu K}{4 k T}
$$

is the dimensionless anisotropy parameter.

\section{APPENDIX B: EXPLICIT REPRESENTATION OF SUBMATRICES IN EQ. (41)}

The submatrices $\mathbf{U}_{4 n-1}, \mathbf{U}_{4 n-2}, \mathbf{U}_{4 n-3}, \mathbf{V}_{4 n}, \mathbf{V}_{4 n-2}, \mathbf{V}_{4 n-3}$ in Eq. (41) are diagonal,

$$
\mathbf{X}_{4 n-1}=\left(\begin{array}{ccccc}
x_{4 n-i,-4\left(n-1+\delta_{i 0}\right)} & 0 & 0 & \cdots & 0 \\
0 & x_{4 n-i,-4\left(n-2+\delta_{i 0}\right)} & x_{4 n-i,-4\left(n-3+\delta_{i 0}\right)} & \cdots & 0 \\
0 & 0 & \vdots & \ddots & 0 \\
\vdots & \vdots & 0 & \cdots & x_{4 n-i, 4\left(n-1+\delta_{i 0}\right)}
\end{array}\right)
$$

$(i=0,1,2,3)$ and have dimension $\left[2\left(n+\delta_{0 i}\right)-1\right] \times\left[2\left(n+\delta_{0 i}\right)-1\right]$. The submatrix $\mathbf{U}_{4 n}$ with the dimension $[2 n+1] \times[2 n$ $-1]$ has the form

$$
\mathbf{U}_{4 n}=\left(\begin{array}{cccc}
0 & 0 & \cdots & 0 \\
u_{4 n,-4 n+4} & 0 & \cdots & 0 \\
0 & u_{4 n,-4 n+8} & \cdots & 0 \\
\vdots & \vdots & \ddots & \ddots \\
0 & 0 & \cdots & u_{4 n, 4 n-4} \\
0 & 0 & \cdots & 0
\end{array}\right)
$$


The submatrix $\mathbf{V}_{4 n-1}$ has dimension $[2 n-1] \times[2 n+1]$ and is defined as

$$
\mathbf{V}_{4 n-1}=\left(\begin{array}{cccccc}
0 & \nu_{4 n-1,-4 n+4} & 0 & \cdots & 0 & 0 \\
0 & 0 & \nu_{4 n-1,-4 n+8} & \cdots & 0 & 0 \\
\ddots & \ddots & \ddots & \ddots & \vdots & \vdots \\
0 & 0 & 0 & \cdots & \nu_{4 n-1,4 n-4} & 0
\end{array}\right)
$$

The elements of the submatrices (B1)-(B3) are given by

$$
u_{n, m}=\left(\frac{n^{2}-m^{2}}{(2 n+1)(2 n-1)}\right)^{1 / 2}, \quad \nu_{n, m}=\left(\frac{(n+1)^{2}-m^{2}}{(2 n+1)(2 n+3)}\right)^{1 / 2}
$$

*Author to whom correspondence should be addressed.

${ }^{1}$ L. Néel, Ann. Geophys. (C.N.R.S.) 5, 99 (1949).

${ }^{2}$ W. F. Brown, Jr., IEEE Trans. Magn. 15, 1196 (1979).

${ }^{3}$ H. B. Braun and H. N. Bertram, J. Appl. Phys. 75, 4609 (1994).

${ }^{4}$ C. P. Bean and J. D. Livingston, J. Appl. Phys. 30, 120S (1959).

${ }^{5}$ A. Aharoni, Phys. Rev. 177, 763 (1969).

${ }^{6}$ Yu. L. Raikher and M. L. Shliomis, Zh. Eksp. Teor. Fiz. 67, 1060 (1974) [Sov. Phys. JETP 40, 526 (1974)].

${ }^{7}$ D. A. Garanin, V. V. Ischenko, and L. V. Panina, Teor. Mat. Fiz. 82, 242 (1990) [Theor. Math. Phys. 82, 169 (1990)].

${ }^{8}$ Yu. L. Raikher and V. I. Stepanov, Zh. Eksp. Teor. Fiz. 102, 1409 (1992) [Sov. Phys. JETP 75, 764 (1992)].

${ }^{9}$ W. T. Coffey, D. S. F. Crothers, Yu. P. Kalmykov, and J. T. Waldron, Phys. Rev. B 51, 15947 (1995).

${ }^{10}$ D. A. Garanin, Phys. Rev. E 54, 3250 (1996).

${ }^{11}$ E. K. Sadykov and A. G. Isavnin, Fiz. Tverd. Tela (Leningrad) 38, 2104 (1996).

${ }^{12}$ Yu. P. Kalmykov and W. T. Coffey, Phys. Rev. B 56, 3325 (1997).

${ }^{13}$ I. Klik and L. Gunther, J. Stat. Phys. 60, 473 (1990).

${ }^{14}$ A. Aharoni, Phys. Rev. B 7, 1103 (1973).

${ }^{15}$ D. A. Smith and F. A. de Rozario, J. Magn. Magn. Mater. 3, 219 (1976).

${ }^{16}$ I. Eisenstein and A. Aharoni, Phys. Rev. B 16, 1278 (1977).
${ }^{17}$ I. Eisenstein and A. Aharoni, Phys. Rev. B 16, 1285 (1977).

${ }^{18}$ I. Klik and L. Gunther, J. Appl. Phys. 67, 4505 (1990).

${ }^{19}$ L. J. Geoghegan, W. T. Coffey, and B. Mulligan, Adv. Chem. Phys. 100, 475 (1997).

${ }^{20}$ W. F. Brown, Jr., Phys. Rev. 130, 1677 (1963).

${ }^{21}$ Yu. L. Raikher and M. I. Shliomis, Adv. Chem. Phys. 87, 595 (1994).

${ }^{22}$ H. Risken, The Fokker-Planck Equation (Springer, Berlin, 1989).

${ }^{23}$ W. T. Coffey, Yu. P. Kalmykov, and J. T. Waldron, The Langevin Equation (World Scientific, Singapore, 1996).

${ }^{24}$ T. L. Gilbert, Phys. Rev. 100, 1243 (1956) (abstract only; full report: Armor Research Foundation Project No. A059, Supplementary Report, May 1, 1956).

${ }^{25}$ R. L. Stratonovich, Conditional Markov Processes and their Application to the Theory of Optimal Control (Elsevier, New York, 1968).

${ }^{26}$ Handbook of Mathematical Functions, edited by M. Abramowitz and I. Stegun (Dover, New York, 1965).

${ }^{27}$ Yu. P. Kalmykov, J. Mol. Liq. 69, 117 (1996).

${ }^{28}$ W. T. Coffey, Adv. Chem. Phys. 103, 259 (1998).

${ }^{29}$ W. T. Coffey, D. S. F. Crothers, J. L. Dormann, Yu. P. Kalmykov, E. C. Kennedy, and W. Wernsdorfer, Phys. Rev. Lett. 80, 5655 (1998). 